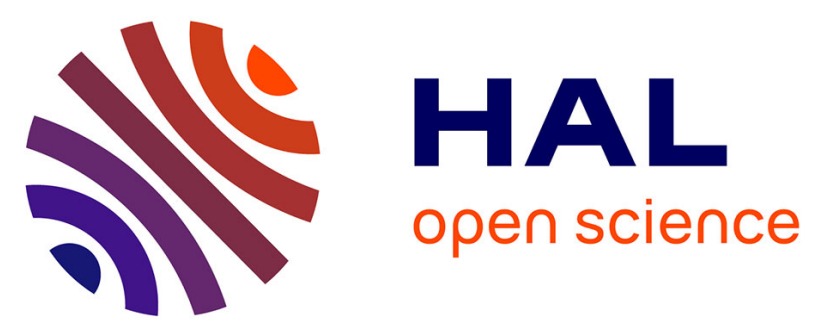

\title{
Nano- and microparticles of gelled oil combining aminoacid-based low molecular weight organogelators and nonionic amphiphilic polysaccharides
}

Carlos Vargas-Gutierrez, Freha Hansali, Guillaume Pickaert, Brigitte Jamart-Gregoire, Alain Durand, Michèle Leonard

\section{To cite this version:}

Carlos Vargas-Gutierrez, Freha Hansali, Guillaume Pickaert, Brigitte Jamart-Gregoire, Alain Durand, et al.. Nano- and microparticles of gelled oil combining aminoacid-based low molecular weight organogelators and nonionic amphiphilic polysaccharides. Colloids and Surfaces A: Physicochemical and Engineering Aspects, 2017, 513, pp.102-109. 10.1016/j.colsurfa.2016.11.036 . hal-02055142

\section{HAL Id: hal-02055142 \\ https://hal.univ-lorraine.fr/hal-02055142}

Submitted on 20 Apr 2019

HAL is a multi-disciplinary open access archive for the deposit and dissemination of scientific research documents, whether they are published or not. The documents may come from teaching and research institutions in France or abroad, or from public or private research centers.
L'archive ouverte pluridisciplinaire HAL, est destinée au dépôt et à la diffusion de documents scientifiques de niveau recherche, publiés ou non, émanant des établissements d'enseignement et de recherche français ou étrangers, des laboratoires publics ou privés. 
Nano- and microparticles of gelled oil combining aminoacid-based low molecular weight organogelators and nonionic amphiphilic polysaccharides (1) Carlos VARGAS-GUTIERREZ ${ }^{1,2}$, Freha HANSALI ${ }^{3}$, Guillaume PICKAERT ${ }^{1,2}$, Brigitte JAMART-GREGOIRE ${ }^{1,2}$, Alain DURAND ${ }^{1,2}$, Michèle LEONARD ${ }^{1,2}$ *
${ }^{1}$ CNRS, LCPM, UMR 7375, Nancy, F-54001, France ${ }^{2}$ Université de Lorraine, LCPM, UMR 7375, Nancy, F-54001, France ${ }^{3}$ Université d'Oran Es-sénia, Oran 31000, Algeria

3

*Corresponding Author: Michèle Léonard, 4 Tel: + $33(0) 3831752$ 76, Fax: + 33 (0)3 83379977

25 Email: Michele.Leonard@univ-lorraine.fr 
29 Nano- and microparticles of gelled oils were prepared using an aminoacid-based low molecular weight organogelator and amphiphilic nonionic polysaccharides as stabilizers. The investigated oils were Tetralin, M810N and M829. The preparation procedure involved organogelator dissolution in hot solvent $\left(150-190{ }^{\circ} \mathrm{C}\right)$ followed by oil-in-water emulsification at $70-80{ }^{\circ} \mathrm{C}$ in liquid state and final cooling step to room temperature to obtain gelled oil particles. The range of particle diameters (sub-micrometric or micrometric) could be adjusted by selecting the emulsification process, sonication or vortex mixing. Using sonication, organogel particles could be also prepared at room temperature with the oil phase in the gel form. The suspensions of gelled oil particles could be centrifuged and fully redispersed by vortex mixing with an increase of particle diameters whose extent depended on the nature of the oil.

\section{Keywords: Organogel, Nanoparticles, Microparticles, Dextran, Aminoacid}




\section{INTRODUCTION}

Low-molecular weight organogelators (LMWO) were defined as small organic molecules (molar mass lower than $3,000 \mathrm{~g} / \mathrm{mol}$ ) with the ability to self-organize in certain organic solvents, giving rise to the formation of fibrous networks that dramatically modify the flow properties of the medium [1]. Self-organization of gelator molecules occurred through the establishment of low energy interactions (for instance hydrogen bonds, ionic interactions, $\pi$ staking, dipole-dipole, solvophobic effects or van der Waals interactions). Among the properties of organogels, two common characteristics were of particular importance. First, the critical gelation concentration (CGC), i.e. the concentration above which gelation was observed at a given temperature. According to the chemical structure of the organogelator, CGC could be reduced down to values well below $0.5 \mathrm{wt} \%$. This was a very interesting point for formulation applications. Second, organogels exhibited thermo-reversibility so that they recovered a liquid-like behavior when temperature exceeded a critical value (which depended on the concentration of organogelator). This property could be used to facilitate the flow of the mixture through pipes or to trigger drug release via viscosity variation for instance. Several studies have been reported about the formation and the mechanical properties of bulk organogels as well as about the links between molecular structure and macroscopic properties [2-7]. Nevertheless, there were much less reports about the use of organogelators in the formulation of disperse systems. Schematically, two types of colloidal dispersions have been reported involving organogels, according to the nature of the gelled phase. On the one hand, water-in-oil emulsions have been prepared in which the oil phase was gelled [8-15]. Gelling continuous oil phase prevented water droplets motion as well as coalescence and thus improved the colloidal stability of the dispersions. On the other hand, the preparation of aqueous suspensions of gelled oil particles has also been reported [16-25]. In that case, gelling disperse phase was closely related to specific applications. Researchers explored different 
ways of controlling the morphology of oil particles on the basis of the properties of the gelator. For instance by inducing an accumulation of the gelator at oil/water interface, capsules were obtained [16]. In another work, the gelator itself was designed so as to serve as chiral template [22]. In most of these formulations, the gelator was 12-hydroxystearic acid (HSA) with minimal concentrations of $0.5 \mathrm{wt} \%$ in the oil.

The design of new LMWO appeared as a promising track in formulation. Indeed innovation in the chemical structure of gelators would be a great opportunity to obtain dispersions with targeted end-use properties like polarity of gelled oil particles, size distribution, colloidal stability in various conditions, temperature reversibility... Some guidelines already existed to relate oil nature and required chemical characteristics of potential gelator candidates using Hansen and Hildebrand solubility parameters [26-32]. Nevertheless there were only a few studies in which colloidal properties of gelled oil dispersions were studied and all of them used HSA as the gelator [17,20].

Some of us reported the design and synthesis of a family of strong LMWO based on aminoacid derivatives $[33,34]$. Gelation of organic solvents was attributed to the formation of an entangled fibrillar network through hydrogen bonds and $\pi-\pi$ stacking interactions $[35,36]$. A detailed analysis of oscillatory rheological properties of these organogels revealed that the macroscopic gel formation resulted from a jamming-induced gelation [37]. When cooling down the organic solution from the liquid state, organogelator molecules formed clusters below a certain temperature. These clusters started crowding when temperature further decreased. When temperature became lower than a critical value called gelation temperature (T ORGANOGEL $_{\text {, }}$, the mechanical behavior of the system changed from liquid to solid-like. Below $\mathrm{T}_{\text {ORGANOGEL }}$ the rheological behavior of the solution was that of a soft solid, with elastic modulus $\left(G^{\prime}\right)$ higher than the loss modulus ( $\left.G^{\prime \prime}\right)$. 
We already reported the preparation of nanometric and micrometric oil-in-water emulsions stabilized by amphiphilic polymers derived from polysaccharides (particularly from dextran, a nonionic bacterial polysaccharide) $[38,39]$. The presence of a dense and thick superficial layer of hydrophilic loops at the surface of oil droplets provided good colloidal stability and offered the possibility of further chemical functionalization thanks to the reactive hydroxyl groups present at the surface. In addition, polysaccharide-covered oil droplets were used as templates for the preparation of polyester nanoparticles via emulsion-solvent evaporation procedure [40].

In that work, we investigated the use of aminoacid-derived gelator molecules for the formulation of gelled oil particle suspensions stabilized by amphiphilic polymers derived from dextran. We particularly focused on the links between the formulation or process variables and the particle size distribution as well as colloidal stability of the suspensions. For the preparation of the particles, we selected three oils comprising two triglycerides currently used in pharmaceutical formulations. The targeted application of the particles was encapsulation of hydrophobic active substances. The presence of dextran chains at the surface of particles was intended for providing colloidal stability as well as opportunity to attach functional groups.

\section{EXPERIMENTAL}

\subsection{Materials}

All chemicals were purchased from Sigma-Aldrich ${ }^{\circledR}$ and used as received. The native dextran, $\mathrm{T} 40^{\circledR}$ sample, had a weight-average molar mass close to $40,000 \mathrm{~g} / \mathrm{mol}$ according to the provider. MilliQ ${ }^{\circledR}$ water was employed for preparing aqueous solutions. The hydrophobically modified dextrans used in that work were synthesized following a previously reported 
procedure [41]. Briefly, dextran $\mathrm{T}_{40}{ }^{\circledR}(10 \mathrm{~g})$ was dissolved in $100 \mathrm{~mL}$ DMSO at room temperature. After $1 \mathrm{~h}$, the required amounts of tetrabutyl ammonium hydroxide (TBAOH) and 1-2 epoxyoctane or 1-2 epoxydodecane were added to the solution. The reaction medium was stirred over $48 \mathrm{~h}$ at room temperature. The crude mixture was dialyzed against a 1:1 (v:v) mixture of deionized water and ethanol, followed by deionized water. The modified dextran was recovered by freeze drying and analyzed by ${ }^{1} \mathrm{H}$ NMR in DMSO $\mathrm{d}_{6}$ in which a few drops of $\mathrm{D}_{2} \mathrm{O}$ were added. The degree of substitution $\tau(\%)$ was defined as the molar ratio (expressed in percents) of attached hydrocarbon groups to the number of glucose repeat units $\left(\tau=100 . y /(x+y)\right.$ see Figure 1). Polymers were named $\operatorname{DexC}_{6}-\tau$ and $\operatorname{DexC}_{10}-\tau$ where $C_{6}$ and $\mathrm{C}_{10}$ referred to the nature of hydrocarbon group. Hydrocarbon groups were assumed to be statistically distributed along polysaccharide backbone.

2.2 Synthesis and structure of aminoacid-based organogelator.

We used L-aminoacid-type gelators with carboxybenzyl $(\mathrm{Z})$ as the amine-protecting group and a naphthalimide moiety (Figure 2). As in previous studies, this molecule was named (L)Phe. The synthesis, characterization and gelation properties of (L)-Phe have been described previously $[32,33,35]$. Briefly, commercial Z-Phe-OH was methylated using $\mathrm{SOCl}_{2}$ and $\mathrm{MeOH}$ in THF. Z-phe-OMe was then reacted with hydrazine in methanol and finally with naphtalic anhydride in toluene.

\subsection{Emulsion formulation and preparation}

Two procedures were designed for preparing suspensions of gelled oil particles (Figure 3). The first procedure (P1) consisted in dissolving (L)-Phe during $1 \mathrm{~h}$ in the solvent at $150-190$ ${ }^{\circ} \mathrm{C}$ and performing emulsification immediately after by sonication or vortex mixing at $70-80$ ${ }^{\circ} \mathrm{C}$, so as to keep the oil phase in a liquid state. Then, oil-in-water emulsion was cooled down 
to room temperature before characterizations. The second procedure $(\mathrm{P} 2)$ comprised the same operations but, after dissolving (L)-Phe at $150-190{ }^{\circ} \mathrm{C}$ in the solvent, the oil phase was cooled down to room temperature, thus taking the form of a gel. Emulsification was then carried out by sonication at room temperature after mixing the liquid aqueous phase with the gelled oil phase. The chemical stability of oils and (L)-Phe during the heating step was checked by comparing ${ }^{1} \mathrm{H}$ NMR spectra before and after the procedure. No difference could be detected for all compounds. Sonication (pulsed mode) was carried out using a Vibracell model 600W (Sonics \& Materials Inc, Danbury, CT). For most experiments hydrophobically modified dextran derivatives, $\operatorname{DexC}_{\mathrm{n}}-\tau$, were used as stabilizers during emulsification step. For comparison, some experiments were carried out where $\operatorname{DexC}_{\mathrm{n}}-\tau$ was replaced by an anionic surfactant, sodium dodecyl sulfate.

\subsection{Determination of average droplet diameter and droplet diameter distribution}

Average hydrodynamic droplet diameters were measured by dynamic light scattering (DLS) at low concentration using a HPPS ${ }^{\circledR}$ from Malvern. Dilution of emulsion samples was carried out with MilliQ water. It was checked that the extent of the dilution did not change significantly the results. The diameter obtained was an average value based on three consecutive measurements for each sample and calculated from the analysis of the autocorrelation function. Taking into account the observed variations between two consecutive measurements, we considered that the obtained average values were known within an interval of $\pm 5 \mathrm{~nm}$. The "polydispersity index" (PDI) provided by HPPS was a reasonable way to appreciate whether the sample size distribution could be considered as monomodal. The PDI was calculated by the software and its value relied on the comparison between the fitted and experimental autocorrelation functions. All samples reported in that work had PDI values lower than 0.35 , which could be considered as characteristic of a 
reasonable agreement of monomodal model with real droplet size distributions of the samples, even if a PDI of 0.35 indicated a rather broad size distribution.

Particle size distributions were also characterized by laser light scattering (Mastersizer $2000^{\odot}$ Malvern Instruments-Cellule: Hydro $2000 \mu$ p). The width of the distribution was quantified by the mean range of particle size named Span (Equation (1)).

$$
\operatorname{Span}=\frac{d(0.9)-d(0.1)}{d(0.5)}
$$

In Equation (1), $\mathrm{d}(0.9), \mathrm{d}(0.5)$, and $\mathrm{d}(0.1)$ were the diameters at $90 \%, 50 \%$ and $10 \%$ cumulative volumes, respectively.

A direct comparison of the measurements made by both techniques (light scattering and DLS) with a suspension of gelled olive oil particles stabilized by $\mathrm{DexC}_{10}-12$ led to the following results: $\quad$ average diameter equal to $171 \mathrm{~nm}$ and PDI equal to 0.24 by DLS, $\mathrm{d}(0.5)$ equal to $177 \mathrm{~nm}$ and Span equal to 2.2 by laser light scattering.

These results showed the consistency of results obtained by both techniques.

2.5 Spectroscopic techniques for the study of chemical structure and hydrogen bond formation Organogels were analysed by Fourier Transform Infra Red spectroscopy to detect the formation of hydrogen bonds between $\mathrm{NH}$ and $\mathrm{CO}$ groups by examination of the variation of the stretching bands i.e. $3500-3200 \mathrm{~cm}^{-1}$ for $\mathrm{NH}$ and $1800-1600 \mathrm{~cm}^{-1}$ for $\mathrm{CO}$.

${ }^{1} \mathrm{H}$ NMR spectra were recorded using a Bruker Avance $300 \mathrm{MHz}$ spectrometer at $25{ }^{\circ} \mathrm{C}$.

\subsection{Rheological measurements}

Oscillatory experiments were performed at imposed shear stress (1 Pa) and $1 \mathrm{~Hz}$ frequency using a parallel plate geometry $(25 \mathrm{~mm}$ diameter $)$ within the linear viscoelastic domain where storage $G$ ' and loss $G$ ” moduli were independent of the stress (Advance Rheometer AR 2000, TA instrument). Then the temperature was increased $\left(1{ }^{\circ} \mathrm{C} / \mathrm{min}\right)$ to determine $\mathrm{T}_{\text {ORGANOGEL }}$. 
2.7 Surface tension measurements

194

195

Surface tension measurements were carried out at $25{ }^{\circ} \mathrm{C}$ using a $\mathrm{K} 20$ surface tensiometer (Krüss easyDyne). Surface tensions were measured using the Lecomte du Noüy ring method. All samples were equilibrated for a sufficient time (15 $\min$ to $1 \mathrm{~h})$ to reach constant readings.

\section{RESULTS AND DISCUSSION}

\subsection{Formulation of oil phase}

The selected oils were one aromatic hydrocarbon (Tetralin) and two aliphatic triglycerides (Miglyol 810N ${ }^{\circledR}$, M810N, and Miglyol 829 ${ }^{\circledR}$, M829). For all oils, 1 wt \% of (L)-Phe was enough to obtain a gelled oil phase at room temperature (Table 1).

A series of experiments was carried out with the aim to roughly evaluate the characteristic times of gelation according to (L)-Phe concentration and nature of the oil. After complete dissolution of (L)-Phe in the oil (at 100 or $150{ }^{\circ} \mathrm{C}$ ), the oil phase was taken out of the heating bath and let cooling. The physical state of the oil phase was visually inspected at regular time intervals turning upside down the vial to evaluate flowing ability. Whatever the solvent used, oil phases containing $2 \mathrm{wt} \%$ (L)-Phe exhibited almost instantaneous gelation after starting the cooling step. For Tetralin and M810N oils, $1 \mathrm{wt} \%$ solutions also exhibited very fast gelation after the beginning of the cooling step (Table 1).

The agreement between qualitative visual observations and quantitative rheological measurements was checked with formulations containing M810N. Oscillatory experiments carried out at $20{ }^{\circ} \mathrm{C}$ with 1 wt $\%$ (L)-Phe dissolved in M810N confirmed the formation of a strong gel (Figure 4a, $G^{\prime}>G^{\prime}$ and very few variation with frequency). Furthermore, temperature variation from $20{ }^{\circ} \mathrm{C}$ to $150{ }^{\circ} \mathrm{C}$ revealed a sharp decrease of both moduli at $\mathrm{T}_{\text {ORGANOGEL }}=85^{\circ} \mathrm{C}$ accompanied by a crossover point above which $G^{\prime}<G^{\prime}$ (Figure $4 \mathbf{b}$ ). 
217 Finally, after reaching $150{ }^{\circ} \mathrm{C}$, the gel sample was let cooling down and both moduli were continuously measured. Storage and loss modulus recovered their maximal respective values after 25 min and $G$ ' became higher than $G$ ”' after about $15 \min$ (Figure 4c).

\subsection{Preparation of gelled oil suspensions}

222 Suspensions of micrometric or sub-micrometric particles of gelled oil were prepared following two procedures (P1 and P2). Previous data (see section 3.1) about kinetics of gelation upon cooling were used to set experimental conditions. For stabilizing oil-in-water emulsions, amphiphilic derivatives of dextran were used (Figure 1). Surface activity and emulsifying properties of these polymers have been reported previously in detail, for the preparation of both micrometric and sub-micrometric emulsions [42-44]. Measurements of the surface tension of solutions of (L)-Phe in Tetralin with concentrations between $2.10^{-4}$ and $2.10^{-1}$ wt $\%$ at $20{ }^{\circ} \mathrm{C}$ revealed no significant difference with that found with Tetralin alone. Thus, we considered that (L)-Phe exhibited no detectable surface active properties and that interfacial tension was controlled by amphiphilic 232 polysaccharides.

233 For all experiments, the stabilizer was dissolved in the aqueous phase before contacting it with the oil phase. We intended to prepare suspensions of gelled oil particles containing about $9 \% \mathrm{v} / \mathrm{v}$ of disperse phase. In previous work we showed that to obtain nanoparticles, a minimum of 5 wt $\%$ (relative to oil phase) of amphiphilic dextran derivative should be loaded 237 in the aqueous phase [42]. Thus we chose to fix the concentration of amphiphilic dextran at 5 $238 \mathrm{~g} / \mathrm{L}$ in the aqueous phase.

239 Comparative experiments were carried out with a widely used anionic surfactant, sodium 240 dodecyl sulfate (SDS). The concentration of SDS was, for the sake of comparison, set to 241 identical value. 
$243 \quad 3.3$ Characterization of particle size distribution of suspensions of gelled particles

244 Suspensions were characterized either by determining average droplet diameter by dynamic

245 light scattering or by using laser granulometry to obtain the full droplet diameter distributions.

246 Gelled Tetralin particle suspensions were first prepared following both P1 and P2 procedures

247 (Figure 3). In case of Tetralin, with or without dissolved (L)-Phe, it was not possible to obtain

248 stable particles with diameters well below $1 \mu \mathrm{m}$ (data not shown). This was attributed to very

249 fast Ostwald ripening [45]. Because of the significant solubility of Tetralin in water (higher

250 than $30 \mathrm{mg} / \mathrm{L}$ at $20^{\circ} \mathrm{C}$ ), stable emulsions with droplet diameters below $1 \mu \mathrm{m}$ were impossible

251 to obtain during a significant time interval. Consequently, we added an ultra hydrophobe oil

252 (hexadecane) in Tetralin, which was a well-known way of slowing down Ostwald ripening

253 [38]. When $5 \% \mathrm{v} / \mathrm{v}$ hexadecane was added into Tetralin, nanoemulsions with average oil

254 diameters around $150 \mathrm{~nm}$ were obtained, either in the absence or in the presence of dissolved

255

(L)-Phe. This demonstrated that despite its insolubility in water (L)-Phe did not allow slowing down of Ostwald ripening contrary to hexadecane. This result was consistent with previous NMR study which showed, in case of a gel formed by (L)-Phe in toluene, that there were no measurable interactions between the supramolecular network and solvent molecules which filled free space within the structure [36]. Regarding the gelation properties, the presence of hexadecane in Tetralin did not prevent the formation of a gel in the same experimental conditions.

When comparing suspensions of gelled particles prepared by procedure P1 with sonication and procedure $\mathrm{P} 2$, we found that average particle diameters were similar. Thus the physical state of the oil phase, liquid or gel, during the emulsification step, had no significant effect on the average particle diameter provided that sonication was used. This result was explained by the high energy provided to the biphasic mixture during the sonication step. Indeed, the 
minimal droplet diameter that could be reached was still below that obtained in the dispersions. Thus the viscosity of the oil phase was not the limiting parameter for these experiments. Additionally, a part of this energy was lost in heat, which could even liquefy the oil phase during sonication, but we had no direct proof of this assumption. Similarly, increasing the concentration of (L)-Phe in Tetralin from 0.3 to $0.7 \mathrm{wt} \%$ did not induce any significant variation in the average particle diameter. When the amount of oil phase was varied between 4.5 and $16 \% \mathrm{v} / \mathrm{v}$, average particle size remained around $150 \mathrm{~nm}$. These results showed that it was possible to vary the amount and the mechanical strength of prepared gelled oil particles while keeping similar particle size distributions (Table 2).

Using either SDS or DexC $10^{-12}$ as the stabilizer, in the presence or in the absence of (L)-Phe in the oil phase, led to similar results (Figure 5). The maximum difference observed in particle/droplet diameter was ca. $40 \mathrm{~nm}$ lower in the case of organogel nanoparticles stabilized by SDS as compared to those stabilized by $\operatorname{DexC}_{10}-12$. Thus, the use of polysaccharide-based stabilizer did not limit the final particle diameter as compared to molecular surfactant.

Because of the formation of hydrogen bonds in the gel state, characteristic FT-IR signals from $\mathrm{NH}$ and $\mathrm{CO}$ groups were evidenced [35]. FT-IR spectra of gels formed from 4 wt \% (L)-Phe solution in Tetralin before and after emulsification in $5 \mathrm{~g} / \mathrm{L} \mathrm{DexC} \mathrm{C}_{10}-12$ aqueous solution and centrifugation were compared (Figure 6). Signals from $\mathrm{NH}$ and $\mathrm{CO}$ were very similar, for both the gel formed by cooling the bulk solution in Tetralin and the gel formed by cooling the oil phase centrifuged from $\mathrm{O} / \mathrm{W}$ emulsions. This experiment confirmed that the gelation phenomenon in Tetralin was similar in bulk and in emulsified state.

The procedure $\mathrm{P} 1$ involving emulsification at $70-80{ }^{\circ} \mathrm{C}$ (Figure 3) was followed in the rest of this work. 
Sub-micrometric particles of gelled oil were obtained using either M810N or M829 and (L)Phe concentrations between 0.5 and $2.0 \mathrm{wt} \%$ in the oil (Table 3). Increasing sonication power and duration allowed displacing particle diameter distribution towards lower diameter and Span values. Particle diameter distributions were similar for both oils. When replacing sonication step by vortex mixing, a much less energetic operation, micrometric suspensions of particles, with high size polydispersity, were obtained with both oils (Table 3). Thus microparticles could be produced with M810N or M829 by adjusting the operating variables of the emulsification step.

As a conclusion, these experiments demonstrated that it was possible to prepare submicrometric and micrometric suspensions of (L)-Phe gelled oil particles covered by dextran chains. By varying (L)-Phe concentration in the oil phase, the mechanical behavior of the gelled core could be modified while maintaining rather unchanged particle diameter distributions.

\subsection{Shelf life of suspensions of gelled oil particles and their stability to centrifugation}

Average particle diameter of Tetralin organogels was monitored over $100 \mathrm{~h}$ and no significant variation could be detected (Figure 5). The same result was obtained with or without (L)-Phe in the oil phase and using SDS or DexC $\mathrm{C}_{10^{-}} 12$ as the stabilizer. Thus suspensions of gelled oil particles exhibited good shelf life.

In the case of suspensions stabilized by $\operatorname{DexC}_{6}-15$, the ability to redisperse in water upon vortex agitation after centrifugation was examined. Whatever the solvent used (Tetralin, M810N and M829) centrifuged particle suspensions could be fully redispersed by vortex agitation. This ability to redisperse after centrifugation was due to efficient stabilization by adsorbed dextran chains which produced steric repulsions between particles. Such 
redispersion ability has been already demonstrated in the case of oil-in-water emulsions stabilized by amphiphilic dextran derivatives $[38,43]$.

Nevertheless, regarding the preservation of the initial particle diameter distribution, we observed rather different results according to the oil used (Table 4). With Tetralin and M829 a limited increase of $\mathrm{d}(0.5)$ was observed. Nevertheless, aggregates were formed (with sizes lower than $100 \mu \mathrm{m}$ ) which led to a significant increase of Span values. In the case of M810N $\mathrm{d}(0.5)$ was increased dramatically and aggregates with diameters above $100 \mu \mathrm{m}$ were formed (Span values increased in most cases). These results showed that despite steric repulsions originating from adsorbed macromolecules, when particles were forced to pack together during centrifugation step, aggregation of particles was limited but not completely avoided, as it had been shown in the case of oil-in-water emulsions and polymeric nanoparticles. The degree of substitution of the dextran derivative was a relevant structural parameter for reducing the extent of aggregation during centrifugation. Previous results had shown that degrees of substitution equal to $15 \%$ for $\mathrm{C}_{6}$ groups and $7 \%$ for $\mathrm{C}_{10}$ groups were sufficient to obtain complete redispersion after lyophilization.[40]

\section{CONCLUSION}

The preparation of nano- and micrometric dextran-covered gelled oil particles was described for the first time. Several oils (Tetralin, M810N and M829) were gelled by an aminoacid derivative. Two procedures for suspensions preparation were designed both involving dissolution of (L)-Phe in oil at $150-190{ }^{\circ} \mathrm{C}$ but differing by the dispersion step. In one procedure, dispersion was prepared by emulsification of hot liquid phases $\left(70-80{ }^{\circ} \mathrm{C}\right)$ while in the other procedure, gelled oil was used and dispersed at room temperature in aqueous phase. The influence of several process parameters and formulation variables was investigated. The use of dextran-based stabilizers led to gelled oil particles with size distributions comparable to 
340 those produced using a common anionic surfactant, SDS. The mechanical strength of the

341 gelled oil core was varied by modifying the concentration of the gelator, which could be done

342 without significant changes in the particle size distribution. Conversely, changing the

343 emulsification step (replacing sonication by vortex mixing) allowed displacing particle

344 diameter distribution from sub-micrometric to micrometric domain. The properties of the

345 gelled oil particles could be adjusted by taking the opportunity of available chemical

346 functionalities: within the gelator molecules and at the surface of the particles with the

347 hydroxyl groups of dextran chains.

348 Current work explores the use of these gelled oil particles for encapsulation and controlled

349 release of active molecules. More specifically, the effect of including another ingredient (the

350 active molecule) in the formulation and in the preparation process of gelled oil particle is 351 investigated in detail.

352

353 


\section{References}

355

356

357

358

359

360

361

362

363

364

365

366

367

368

369

370

371

372

373

374

375

376

377

378

379

[1] P. Terech, R.G. Weiss, Low molecular mass gelators of organic liquids and the properties of their gels, Chem. Rev. 97 (1997) 3133-3159.

[2] N.M. Sangeetha, U. Maitra, Supramolecular gels: Functions and uses, Chem. Soc. Rev. 34 (2005) 821-836.

[3] F.R. Lupi, D. Gabriele, V. Greco, N. Baldino, L. Seta, B. de Cindio, A rheological characterisation of an olive oil/fatty alcohols organogel, Food Res. Int. 51 (2013) 510-517.

[4] A.R. Patel, M. Babaahmadi, A. Lesaffer, K. Dewettinck, Rheological profiling of organogels prepared at critical gelling concentrations of natural waxes in a triacylglycerol solvent, J. Agric. Food Chem. 63 (2015) 4862-4869.

[5] J. Daniel, R. Rajasekharan, Organogelation of plant oils and hydrocarbons by long-chain saturated FA, fatty alcohols, wax esters, and dicarboxylic acids, J. Am. Oil Chem. Soc. 80 (2003) 417 421.

[6] A.J. Wright, A.G. Marangoni, Time, temperature, and concentration dependence of ricinelaidic acid-canola oil organogelation, J. Am. Oil Chem. Soc. 84 (2007) 3-9.

[7] M. Burkhardt, L. Noirez, M. Gradzielski, Organogels based on 12-hydroxy stearic acid as a leitmotiv: Dependence of gelation properties on chemical modifications, J. Colloid Interface Sci. 466 (2016) 369-376.

[8] J.F. Toro-Vazquez, R. Mauricio-Perez, M.M. Gonzalez-Chavez, M. Sanchez-Becerril, J.d.J. Ornelas-Paz, J.D. Perez-Martinez, Physical properties of organogels and water in oil emulsions structured by mixtures of candelilla wax and monoglycerides, Food Res. Int. 54 (2013) 1360-1368.

[9] S. Murdan, B. van den Bergh, G. Gregoriadis, A.T. Florence, Water-in-sorbitan monostearate organogels (water-in-oil gels), J. Pharm. Sci. 88 (1999) 615-619.

[10] C. Stubenrauch, R. Tessendorf, A. Salvati, D. Topgaard, T. Sottmann, R. Strey, I. Lynch, Gelled polymerizable microemulsions. 2. Microstructure, Langmuir 24 (2008) 8473-8482. 

microemulsions. 1. Phase behavior Langmuir 23 (2007) 7730-7737.

[12] C. Holtze, K. Landfester, M. Antonietti, A novel route to multiphase polymer systems containing nano-droplets: Radical polymerization of vinylic monomers in gelled water-in-oil miniemulsions, Macromol. Mater. Eng. 290 (2005) 1025-1028.

[13] K.Q. Liu, P.L. He, Y. Fang, Progress in the studies of low-molecular mass gelators with unusual properties, Sci. China Chem. 54 (2011) 575-586.

[14] N.E. Hughes, A.G. Marangoni, A.J. Wright, M.A. Rogers, J.W.E. Rush, Potential food applications of edible oil organogels, Trends Food Sci. Technol. 20 (2009) 470-480.

[15] P. Jing, J.L. Yan, X.Q. Cai, J. Liu, B.L. Hu, Y. Fang, Solvent-induced molecular gel formation at room temperature and the preparation of related gel-emulsions, Sci. China Chem. 56 (2013) 982391991.

392 [16] A.R. Patel, C. Remijn, P.C.M. Heussen, R. den Adel, K.P. Velikov, Novel low-molecular393 weight gelator-based microcapsules with controllable morphology and temperature responsiveness, 394 ChemPhysChem 14 (2013) 305-310.

395 [17] P. Kirilov, L. Lukyanova, S. Franceschi-Messant, V. Perier, E. Perez, I. Rico-Lattes, A new 396 type of colloidal dispersions based on nanoparticles of gelled oil, Colloids Surf. A 328 (2008) 1-7.

397 [18] P. Kirilov, F. Gauffre, S. Franceschi-Messant, E. Perez, I. Rico-Lattes, Rheological 398 characterization of a new type of colloidal dispersion based on nanoparticles of gelled oil, J. Phys. 399 Chem. B 113 (2009) 11101-11108.

400 [19] J. Zou, D.S. Frost, L.L. Dai, Effects of gelator 12-hydroxystearic acid (12-HSA) on ionic 401 liquid based Pickering emulsions, Colloids Surf. A 414 (2012) 477-485.

402 [20] P. Kirilov, S. Rum, E. Gilbert, L. Roussel, D. Salmon, R. Abdayem, C. Serre, C. Villa, M. 403 Haftek, F. Falson, F. Pirot, Aqueous dispersions of organogel nanoparticles - potential systems for 404 cosmetic and dermo-cosmetic applications, Int. J. Cosmetic Sci. 36 (2014) 336-346. 
Lattes, A.C. Tedesco, Gelled oil particles: A new approach to encapsulate a hydrophobic

407

408

409

410

411

412

413

414

415

416

417

418

419

420

421

422

423

424

425

426

427

428

429

metallophtalocyanine, J. Colloid Interface Sci. 401 (2013) 155-160.

[22] Y. Sun, L. Jin, H. Wang, Y. Yang, Polymerized organogel particles formed and imprinted by chiral gelators and their selective adsorption for phenylalanine racemates, Soft Matter 7 (2011) 348350.

[23] S.S. Sagiri, V.K. Singh, I. Banerjee, K. Pramanik, P. Basak, K. Pal, Core-shell-type organogel-alginate hybrid microparticles: A controlled delivery vehicle, Chem. Eng. J. 264 (2015) $134-145$.

[24] S.S. Sagiri, V.K. Singh, S. Kulanthaivel, I. Banerjee, P. Basak, M.K. Battachrya, K. Pal, Stearate organogel-gelatin hydrogel based bigels: Physicochemical, thermal, mechanical characterization and in vivo drug delivery applications, J. Mech. Behavor Biomed. Mater. 43 (2015) 117.

[25] M.R. Zahi, P. Wan, H. Liang, Q. Yuan, Formation and stability of D-limonene organogelbased nanoemulsion prepared by a high-pressure homogenizer, J. Agric. Food Chem. 62 (2014) 12563-12569.

[26] M. Raynal, L. Bouteiller, Organogel formation rationalized by Hansen solubility parameters, Chem Commun. 47 (2011) 8271-8273.

[27] J. Gao, S. Wu, M.A. Rogers, Harnessing Hansen solubility parameters to predict organogel formation, J. Mater. Chem. 22 (2012) 12651-12658.

[28] Y. Wu, S. Wu, G. Zou, Q. Zhang, Solvent effect on structure, photoresponse and speed of gelation of a dicholesterol-linked azobenzene organogel, Soft Matter 7 (2011) 9177-9183.

[29] L. Feng, K.A. Cavicchi, Investigation of the relationships between the thermodynamic phase behavior and gelation behavior of a series of tripodal trisamide compounds, Soft Matter 8 (2012) 6483-6492. 

component hydrogels of melamine with di(2-ethylhexyl)phosphoric acid, Soft Matter 9 (2013) 30574323062.

433 [31] J. Bonnet, G. Suissa, M. Raynal, L. Bouteiller, Organogel formation rationalized by Hansen solubility parameters: dos and don'ts, Soft Matter 10 (2014) 3154-3160.

[32] P. Curcio, F. Allix, G. Pickaert, B. Jamart-Grégoire, A favorable, narrow, $\delta_{h}$ Hansen parameter domain for gelation of low-molecular-weight amino acid derivatives, Chem. Eur. J. 17 (2011) 1360313612.

[33] N. Brosse, D. Barth, B. Jamart-Grégoire, A familiy of strong low-molecular-weight organogelators based on aminoacid derivatives, Tetrahedron Lett. 45 (2004) 9521-9524.

[34] Q.N. Pham, N. Brosse, C. Frochot, D. Dumas, A. Hocquet, B. Jamart-Grégoire, Influence of the gelator structure and solvent on the organisation and chirality of self-assembling fibrillar networks, New J. Chem. 32 (2008) 1131-1139.

[35] F. Allix, P. Curcio, Q.N. Pham, G. Pickaert, B. Jamart-Grégoire, Evidence of intercolumnar $\pi$ $\pi$ stacking interactions in amino-acid-based low-molecular-weight organogels, Langmuir 26 (2010) 16818-16827.

[36] M. Yemloul, E. Steiner, A. Robert, S. Bouguet-Bonnet, F. Allix, B. Jamart-Grégoire, D. Canet, Solvent dynamical behavior in an organogel phase as studied by NMR relaxation and diffusion experiments, J. Phys. Chem. B 115 (2011) 2511-2517.

[37] D. Collin, R. Covis, F. Allix, B. Jamart-Grégoire, P. Martinoty, Jamming transition in solutions containing organogelator molecules of amino-acid type: rheological and calorimetry experiments, Soft Matter 9 (2013) 2947-2958.

[38] E. Rotureau, E. Marie, M. Leonard, E. Dellacherie, T.A. Camesano, A. Durand, From polymeric surfactants to colloidal systems (2): Preparation of colloidal dispersions, Colloids Surf. A 288 (2006) 62-70.

[39] R. Covis, C. Baravian, E. Marie, A. Durand, Kinetics of formation of polysaccharide-covered micrometric oil droplets under mechanical agitation, Colloids Surf. A 466 (2015) 92-99. 
457 [40] C. Rouzes, M. Leonard, A. Durand, E. Dellacherie, Influence of polymeric surfactants on the 458 properties of drug-loaded PLA nanospheres, Colloids Surf. B 32 (2003) 125-135.

459 [41] E. Rotureau, C. Chassenieux, E. Dellacherie, A. Durand, Neutral polymeric surfactants 460 derived from dextran: A study of their aqueous solution behavior, Macromolecular Chemistry and 461 Physics 206 (2005) 2038-2046.

462 [42] E. Rotureau, M. Leonard, E. Marie, E. Dellacherie, T.A. Camesano, A. Durand, From 463 polymeric surfactants to colloidal systems (1): Amphiphilic dextrans for emulsion preparation, 464 Colloids Surf. A 288 (2006) 131-137.

465 [43] R. Covis, J. Desbrieres, E. Marie, A. Durand, Dilational rheology of air/water interfaces 466 covered by nonionic amphiphilic polysaccharides. Correlation with stability of oil-in-water emulsions, 467 Colloids Surf. A 441 (2014) 312-318.

468 [44] R. Covis, C. Baravian, E. Marie, A. Durand, Kinetics of formation of polysaccharide-covered 469 micrometric oildroplets under mechanical agitation, Colloids Surf. A 466 (2015) 92-99.

470 [45] I.G. Economou, J.L. Heidman, C. Tsonopoulos, Mutual Solubilities of Hydrocarbons and 471 Water: III. 1-Hexene, 1-Octene, C10-C12 Hydrocarbons, AIChE J. 43 (1997) 535-546. 
Table 1: Physical state of (L)-Phe solutions in various oils after complete dissolution during 1

$476 \mathrm{~h}$ at $100{ }^{\circ} \mathrm{C}$ (for Tetralin $+5 \% \mathrm{v} / \mathrm{v}$ hexadecane) or $150{ }^{\circ} \mathrm{C}$ (for M810N and M829) and

477 beginning cooling at room temperature (initial time). Qualifications of "liquid (L)", "gel (G)"

478 and "mixtures of liquid and gel $(\mathrm{L}+\mathrm{G})$ " were attributed on the basis of visual observations.

479

Time after withdrawal from the heating bath (h)

$\begin{array}{llllllll}\text { (L)-Phe } & & & & & & & \\ (\text { wt } \%) & 0.25 & 0.5 & 1 & 2 & 3 & 4 & 24\end{array}$

\begin{tabular}{|c|c|c|c|c|c|c|c|c|c|}
\hline \multirow{3}{*}{ 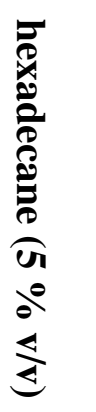 } & \multirow{3}{*}{$\begin{array}{l}\vec{Q} \\
\overrightarrow{7} \\
+ \\
+\end{array}$} & 0.3 & $\mathrm{~L}$ & $\mathrm{~L}$ & $\mathrm{~L}+\mathrm{G}$ & $\mathrm{L}+\mathrm{G}$ & $\mathrm{L}+\mathrm{G}$ & $\mathrm{L}+\mathrm{G}$ & G \\
\hline & & 1.0 & $\mathrm{G}$ & $\mathrm{G}$ & $\mathrm{G}$ & $\mathrm{G}$ & $\mathrm{G}$ & $\mathrm{G}$ & G \\
\hline & & 2.0 & $\mathrm{G}$ & $\mathrm{G}$ & $\mathrm{G}$ & $\mathrm{G}$ & $\mathrm{G}$ & $\mathrm{G}$ & G \\
\hline \multirow{2}{*}{\multicolumn{2}{|c|}{ 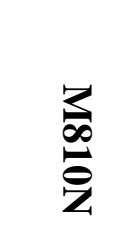 }} & 0.5 & $\mathrm{~L}$ & $\mathrm{~L}$ & $\mathrm{~L}$ & $\mathrm{~L}$ & $\mathrm{~L}$ & $\mathrm{~L}$ & G \\
\hline & & 1.0 & $\mathrm{G}$ & G & $\mathrm{G}$ & $\mathrm{G}$ & $\mathrm{G}$ & $\mathrm{G}$ & G \\
\hline \multirow{3}{*}{\multicolumn{2}{|c|}{ 歺 }} & 0.5 & $\mathrm{~L}$ & $\mathrm{~L}$ & $\mathrm{~L}$ & $\mathrm{~L}$ & $\mathrm{~L}$ & $\mathrm{~L}$ & $\mathrm{~L}$ \\
\hline & & 1.0 & $\mathrm{~L}$ & $\mathrm{~L}$ & $\mathrm{~L}$ & $\mathrm{~L}$ & $\mathrm{~L}$ & $\mathrm{~L}$ & G \\
\hline & & 2.0 & $\mathrm{~L}+\mathrm{G}$ & $\mathrm{G}$ & $G$ & $G$ & $G$ & $\mathrm{G}$ & G \\
\hline
\end{tabular}

480

481 
Table 2: Average particle diameter measured by dynamic light scattering for suspensions of 483 gelled Tetralin (with $5 \% \mathrm{v} / \mathrm{v}$ hexadecane) prepared following procedure $\mathrm{P} 1$ or $\mathrm{P} 2$ and 484 containing various amounts of (L)-Phe in the oil phase. The stabilizer was DexC $\mathrm{C}_{10}-12$ at $5 \mathrm{~g} / \mathrm{L}$ 485 in water and the weight fraction of dispersed phase was 9 wt $\%$.

486

\begin{tabular}{|c|c|c|}
\hline $\begin{array}{c}\text { (L)-Phe } \\
(\mathbf{w t} \%)\end{array}$ & Procedure & $\begin{array}{c}\text { Average particle diameter } \\
(\mathbf{n m})\end{array}$ \\
\hline 0.3 & $\mathrm{P} 1$ & 174 \\
\hline 0.3 & $\mathrm{P} 2$ & 155 \\
\hline 0.5 & $\mathrm{P} 2$ & 148 \\
\hline 0.7 & $\mathrm{P} 2$ & 134 \\
\hline
\end{tabular}

487 
Table 3: Particle diameter distribution determined by laser granulometry for suspension of gelled oil particles prepared following procedure P1.

The stabilizer was $\operatorname{DexC}_{6}-15$ at $5 \mathrm{~g} / \mathrm{L}$ in water and the weight fraction of dispersed phase was 9 wt $\%$.

\begin{tabular}{|c|c|c|c|c|}
\hline \multirow{3}{*}{ Oil } & (L)-Phe & & $\mathrm{d}(\mathbf{0 . 5})$ & \\
\hline & (wt \%) & Emulsification & $(\mu \mathrm{m})$ & Span \\
\hline & 0.5 & Sonication (power $30 \mathrm{~W}, 60 \mathrm{~s}$ ) & 0.41 & 30.3 \\
\hline \multirow{4}{*}{ M810N } & 0.5 & \multirow[b]{2}{*}{ Sonication (power $60 \mathrm{~W}, 120 \mathrm{~s}$ ) } & 0.18 & 3.0 \\
\hline & 1.0 & & 0.19 & 3.8 \\
\hline & 0.5 & \multirow[b]{2}{*}{ Vortex (5 min) } & 9.15 & 9.9 \\
\hline & 1.0 & & 5.37 & 7.0 \\
\hline \multirow{4}{*}{ M829 } & 1.0 & \multirow[b]{2}{*}{ Sonication (power $60 \mathrm{~W}, 120 \mathrm{~s}$ ) } & 0.21 & 4.4 \\
\hline & 2.0 & & 0.22 & 5.0 \\
\hline & 1.0 & \multirow{2}{*}{ Vortex (5 min) } & 5.14 & 23.6 \\
\hline & 2.0 & & 0.69 & 6.5 \\
\hline
\end{tabular}




\begin{tabular}{|c|c|c|c|c|c|}
\hline & \multicolumn{3}{|c|}{ After preparation (24 h) } & \multicolumn{2}{|c|}{ After centrifugation } \\
\hline Oil & $\begin{array}{l}\text { (L)-Phe } \\
(\text { wt \%) }\end{array}$ & $\begin{array}{c}\mathrm{d}(0.5) \\
(\mu \mathrm{m})\end{array}$ & Span & $\begin{array}{c}\mathrm{d}(0.5) \\
(\mu \mathrm{m})\end{array}$ & Span \\
\hline Tetralin + $5 \%$ v/v hexadecane & 1.0 & 0.16 & 5.5 & 0.24 & 12.2 \\
\hline & 0.5 & 0.18 & 3.0 & 5.21 & 7.9 \\
\hline \multirow[t]{3}{*}{ M810N } & 1.0 & 0.19 & 3.8 & 2.04 & 9.6 \\
\hline & 1.0 & 5.37 & 7.0 & 13.31 & 4.9 \\
\hline & 1.0 & 0.21 & 4.4 & 0.27 & 52.6 \\
\hline M829 & 2.0 & 0.22 & 5.0 & 0.27 & 28.9 \\
\hline
\end{tabular}


Figure 1: Chemical structure of amphiphilic dextran derivatives, $\operatorname{DexC}_{6}-\tau$ and $\operatorname{DexC}_{10}-\tau$. The

501

502

503

504

505

506

507

508

509

510

511

512

513

514

515

516

517

518

519

520

degree of substitution was defined by $\tau=100 \times y /(x+y)$. The linear aliphatic hydrocarbon group $\mathrm{R}$ was $-\mathrm{C}_{6} \mathrm{H}_{13}$ and $-\mathrm{C}_{10} \mathrm{H}_{21}$ for $\mathrm{C}_{6}$ and $\mathrm{C}_{10}$ series, respectively.

Figure 2: Chemical structure of (L)-Phe organogelator.

Figure 3: Experimental procedures P1 and P2 followed for preparing suspensions of gelled oil particles. For details see text.

Figure 4: Rheological (oscillatory) measurements with $1 \mathrm{wt} \%$ (L)-Phe solution in M810N. Storage (G') and loss modulus (G') at $20{ }^{\circ} \mathrm{C}$ as a function of frequency (a). Variation of storage and loss modulus with temperature (b). Variation of storage and loss modulus with cooling time after reaching $150^{\circ} \mathrm{C}(\mathrm{c})$.

Figure 5: Average particle diameter determined by dynamic light scattering of 0.3 wt $\%$ (L)Phe Tetralin organogel suspension (bold symbols) or Tetralin emulsion (open symbols) prepared by procedure P1. Tetralin contained $5 \% \mathrm{v} / \mathrm{v}$ hexadecane in all cases. The weight fraction of organic disperse phase was $9 \mathrm{wt} \%$. The stabilizer was SDS (circles) or $\mathrm{DexC}_{10^{-}} 12$ (squares). Time $=0 \mathrm{~h}$ corresponded to measurements carried out immediately after preparation. Lines are guide for the eye. 
521 Figure 6: Comparison of IR bands from NH (left) and CO (right) groups for 4 wt \% gels of

522 (L)-Phe in Tetralin in bulk (red) or in particles recovered after centrifugation of an aqueous 523 suspension (blue).

524

525

526 
Figure 1

528

529

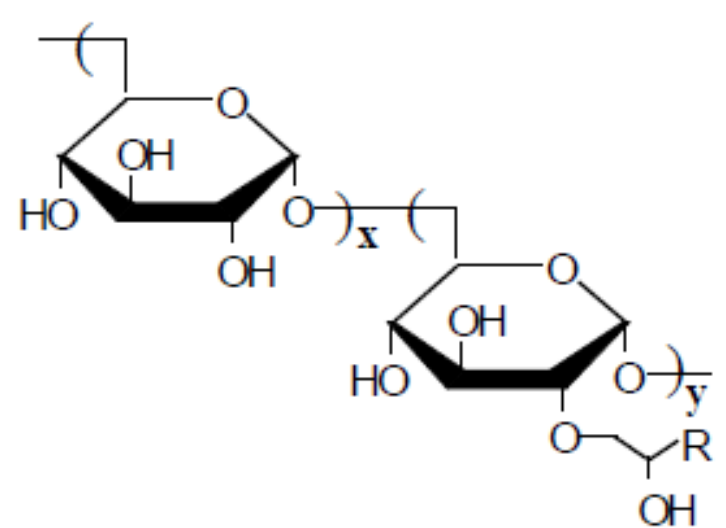

530

531 


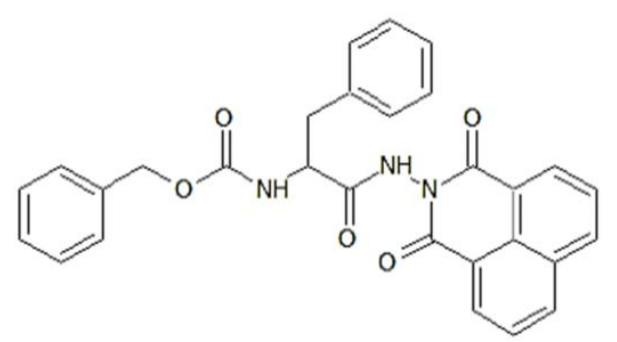

533

534

535 
Figure 3

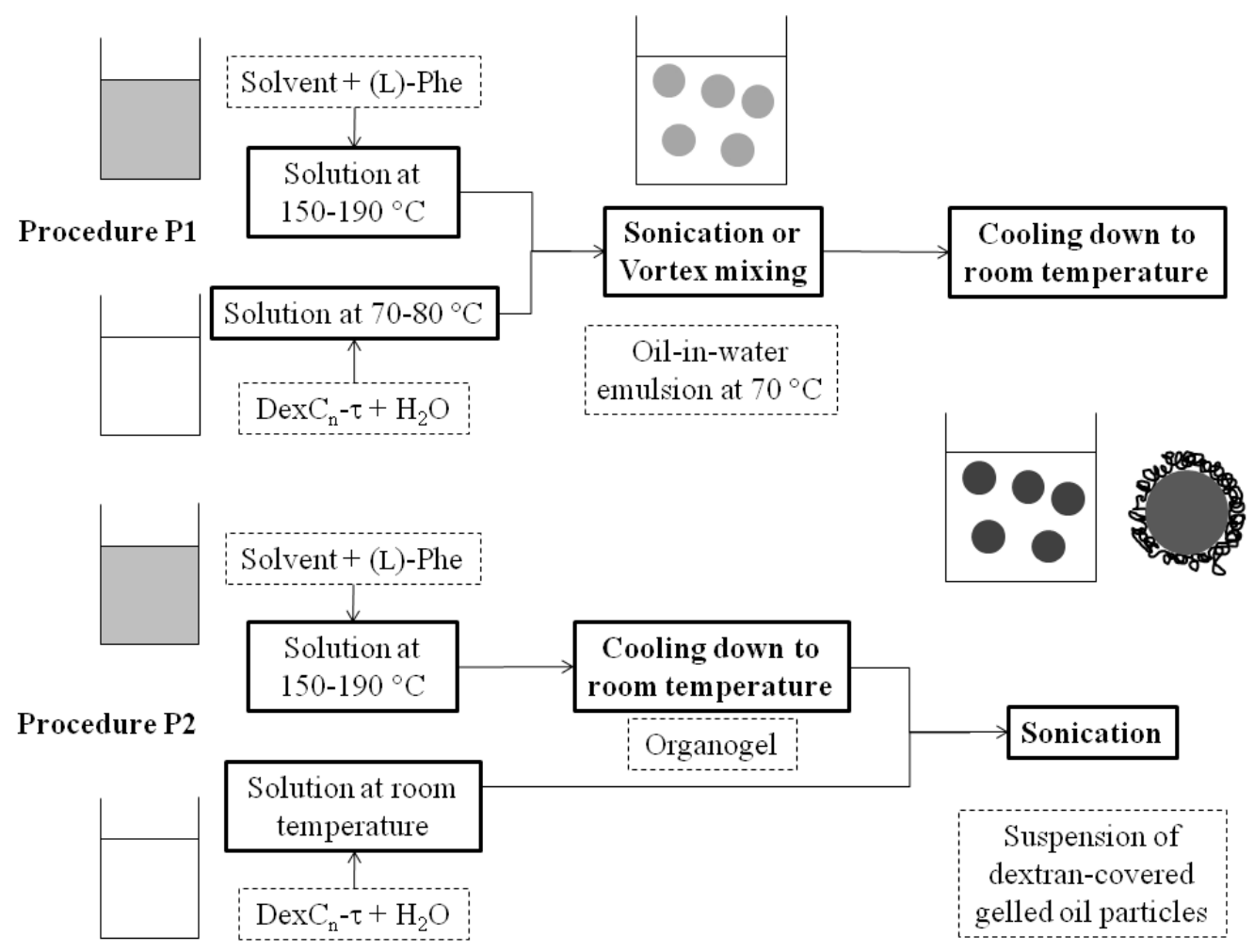


542

\section{a)}

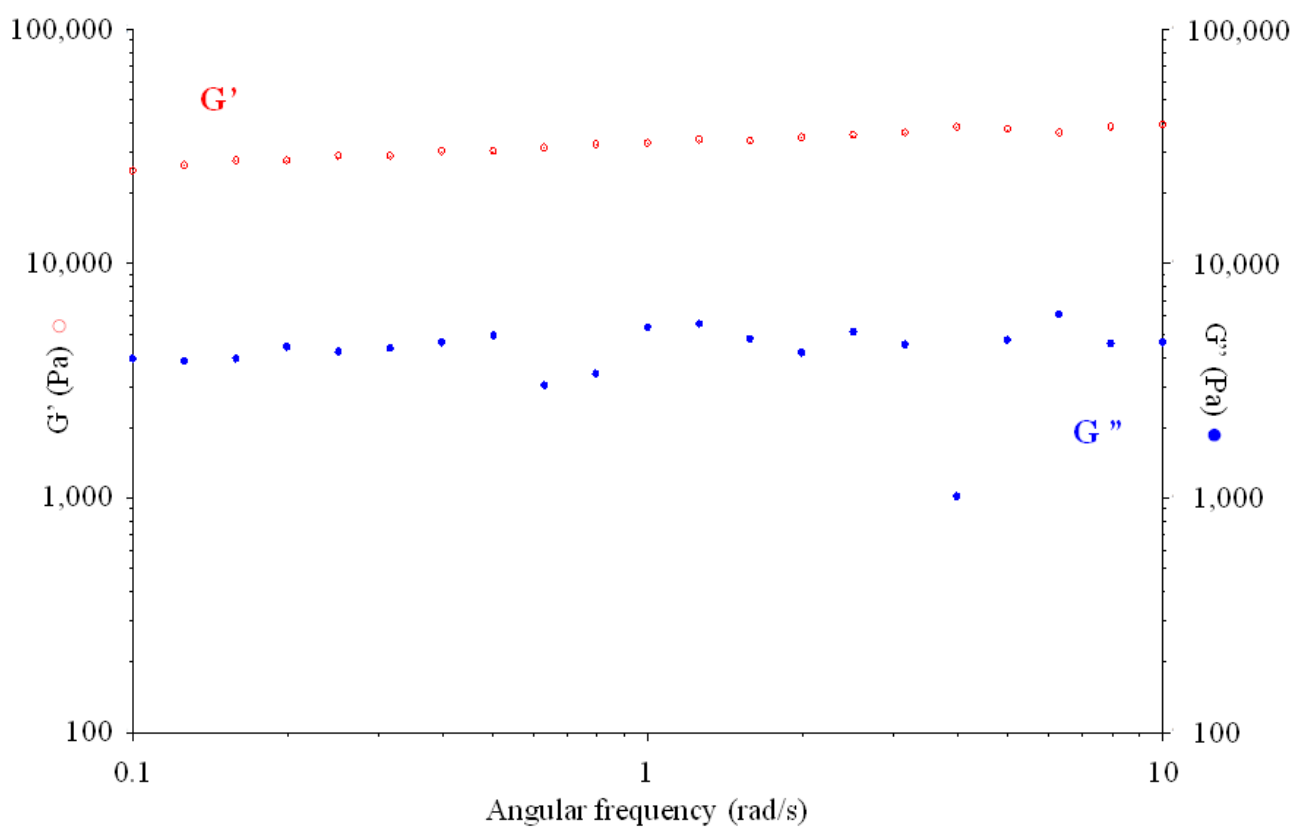

543

544

545

546 
548

b)

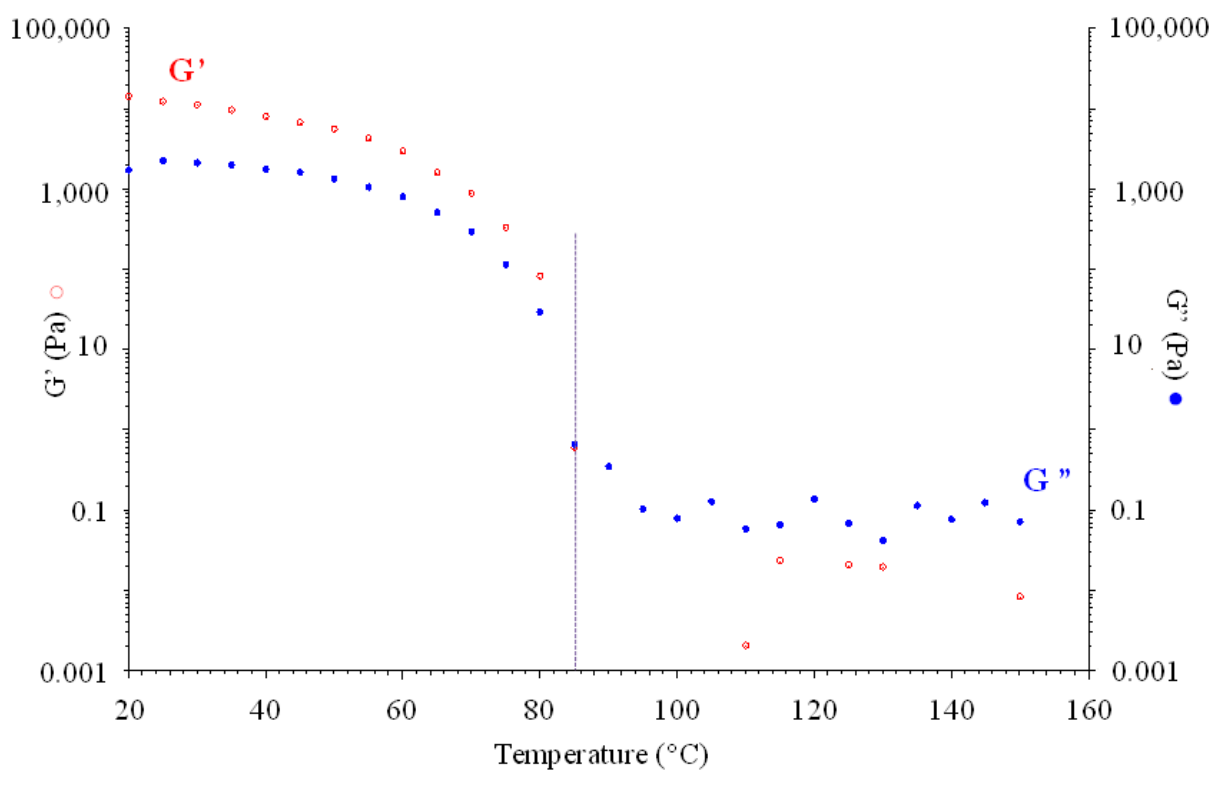

549

550

551 
c)

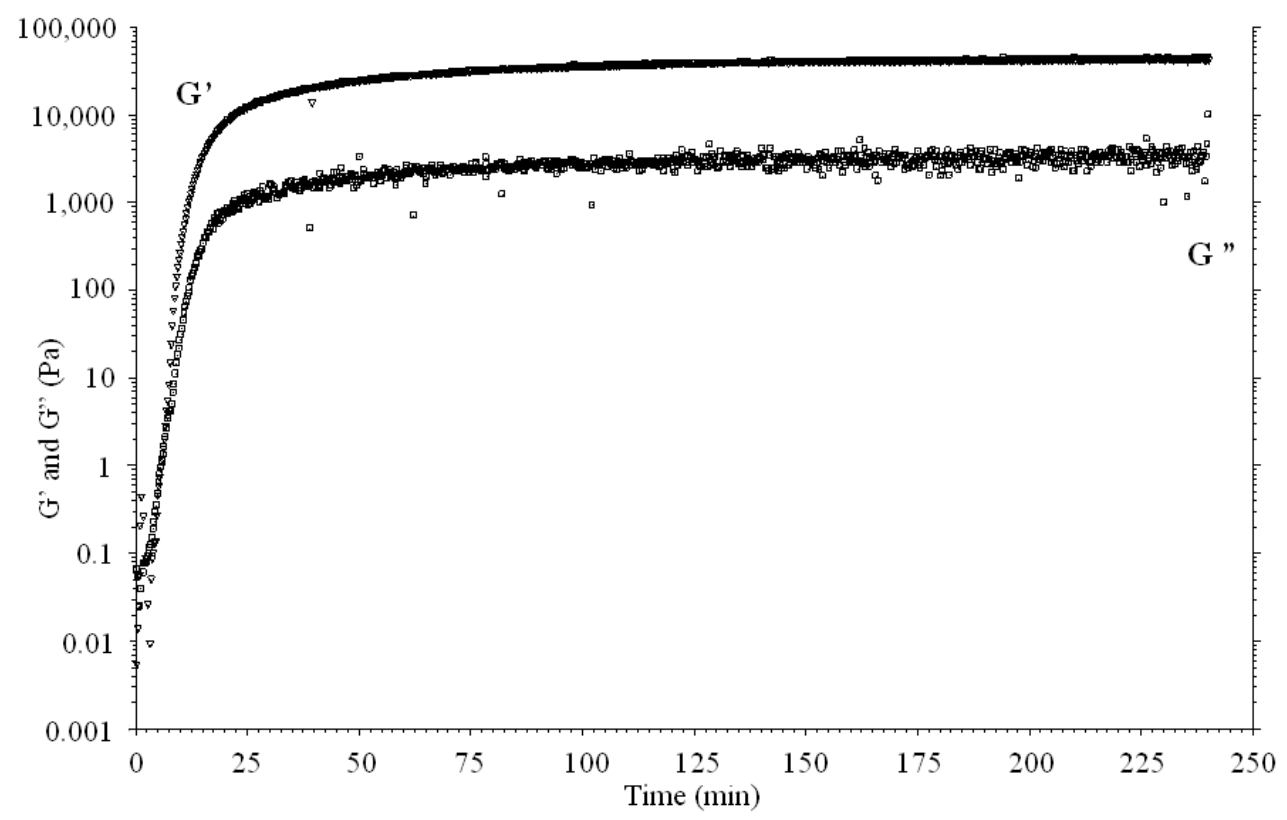

554

555

556 
558

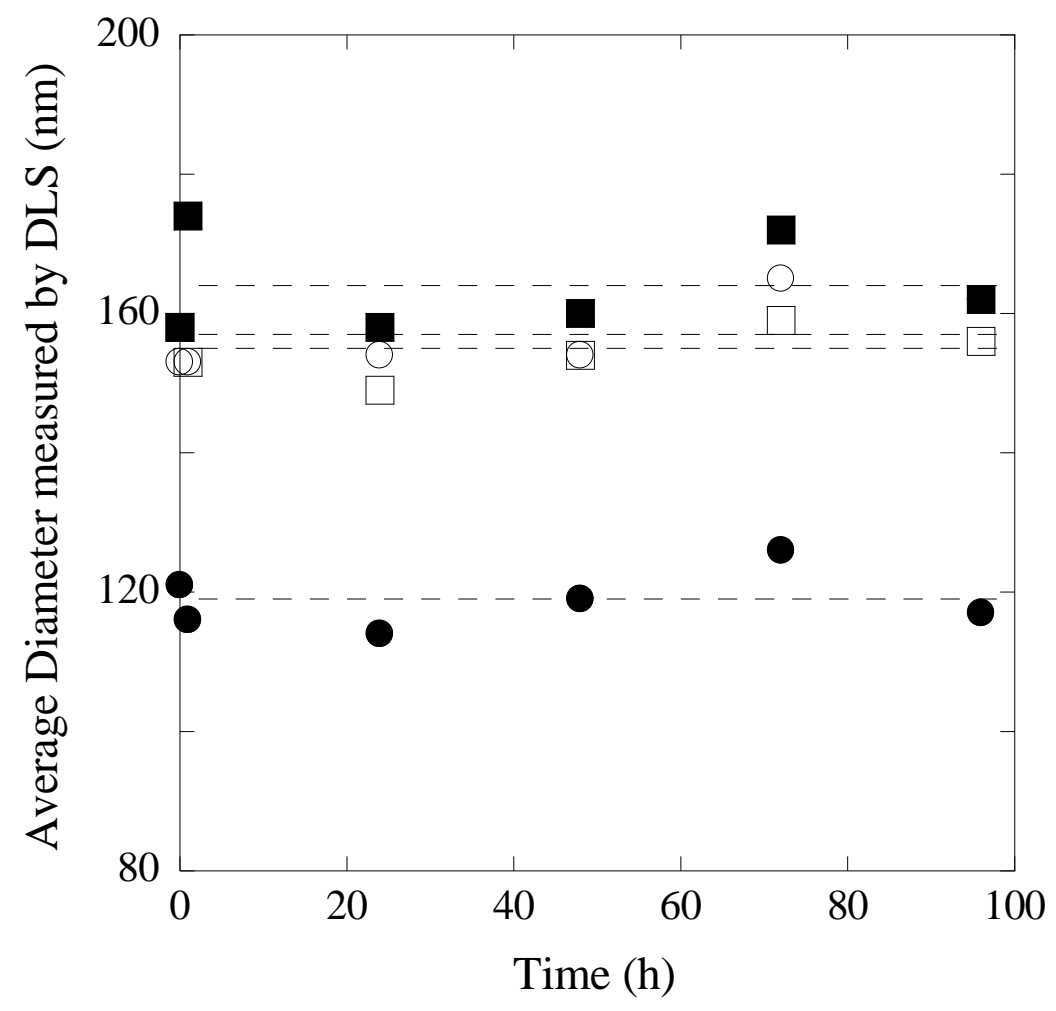

560

561

562

563 
565
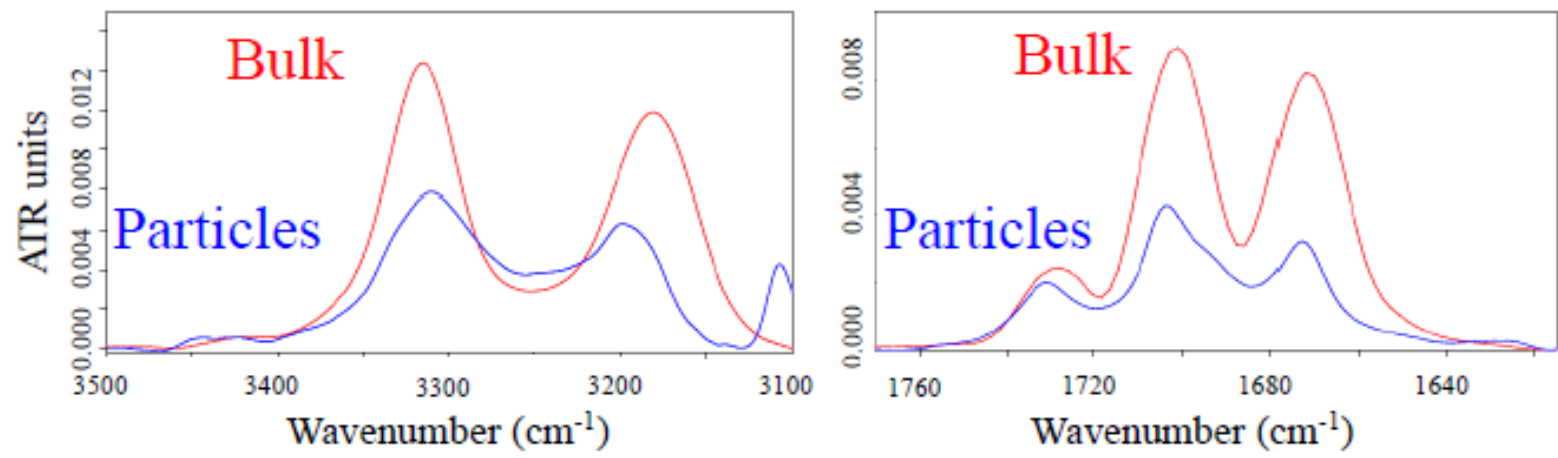

566

567 